\title{
SIMULACION DISCRETA APLICADA AL RUTEO INTRAURBANO
}

\section{DISCRETE SIMULATION APPLIED TO INTRAURBAN ROUTE}

\author{
Javier Darío Fernández Ledesma ${ }^{1, *}$, Laura Lotero Vélez² \\ Laura Vanessa Gutiérrez Arboleda ${ }^{3}$, José David Morales ${ }^{3}$, Jaime Antero Arango Marín ${ }^{4}$
}

\begin{abstract}
RESUMEN
Esta Investigación buscó disminuir los altos costos y las ineficiencias a nivel logístico en el transporte terrestre de mercancías. Para lograrlo, inicialmente se tomaron los datos arrojados por las herramientas tecnológicas empleadas por una compañía en cuatro zonas logísticas, determinando un modelo de simulación discreta con las variables críticas del problema con promodel (statfit): tiempo de cargue, tiempo de recorrido y tiempo de descargue. Los resultados arrojaron que la simulación reduce los tiempos totales en el transporte terrestre hasta en un $29,66 \%$. El modelo de simulación permite tomar decisiones efectivas y eficientes a nivel logístico, a la hora de programar las rutas de entrega y la distribución de las mercancías.
\end{abstract}

Palabras Clave:Logística, distribución, simulación, promodel, transporte terrestre,Medellín.

\section{ABSTRACT}

This research sought to reduce the high costs and inefficiencies at a logistic level in the land transport of goods. To achieve this, the data obtained by the technological tools used by a company in four logistic zones were initially taken, determining a discrete simulation model with the critical variables of the problem with promodel (statfit): loading time, travel time and time of Download The results showed that the simulation reduces the total time in land transport by up to $29,66 \%$. The simulation model allows effective and efficient decisions to be made at the logistics level, when programming the delivery routes and the distribution of the goods.

Keywords:logistics, trucking, simulation, promodel, land transport, Medellin.

\footnotetext{
1 Universidad Pontificia Bolivariana, Facultad de Ingenieria Industrial, Medellin, Colombia, orcid.org/ 0000-0002-6219-2955.

${ }^{2}$ Universidad Pontificia Bolivariana, Facultad de Ingeniería Industrial, Medellin, Colombia, orcid.org/0000-0002-6537-3276.

${ }^{3}$ Universidad Pontificia Bolivariana, Facultad de Ingeniería Industrial, Medellín, Colombia.

${ }^{4}$ Universidad Católica de Manizales, Facultad de Ingeniería Industrial, Manizales, Colombia, orcid.org/0000-0003-0626-6013
}

•Autor para correspondencia: javier.fernandez@upb.edu.co. 


\section{INTRODUCCIÓN}

A nivel ubano la distribución de mercancías se ha vuelto cada vez más compleja, debido a los problemas de movilidad y de disponibilidad de espacios.

De acuerdo con Lamb et al. (2002), la logística es "el proceso de gestionar estratégicamente el flujo eficiente y el almacenamiento de materias primas, existencias en proceso y productos terminados desde el punto de origen hasta el punto de consumo".

Ferrel et al. (2004), define a la logística como "una función operativa importante que incluye todas las actividades necesarias para la obtención y gestión de materias primas y componentes, así como el manejo de los productos terminados, su embalaje y su distribución a los clientes".

Carranza \& Sabria (2005) entienden la logística como "el proceso responsable de planificar, implementar y controlar de manera eficiente y económica el flujo y almacenamiento de materias primas, inventarios en proceso, productos terminados e información vinculada a ellos desde el punto de origen hasta el punto de consumo con el fin de adaptarse a los requerimientos del cliente".

Y finalmente, Robuste (2005) define la logística como la ciencia que estudia la forma en que los bienes, las personas o la información superan el tiempo y la distancia de manera eficiente.

Específicamente, en logística de transporte, según Caplice (2006), podría entenderse por la optimización de rutas, todas aquellas acciones que contribuyen a la mejora de la función de distribución, ya sea en términos de nivel de servicio, mejoras de calidad, reducción de costos, etc.

Algunos de los trabajos más representativos estudiados en modelos de optimización logística durante la década anterior en Latinoamérica son: modelos de observación de carga (MinTransporte, 2009); el modelo de transporte de cuatro etapas (MinPlanificación y Cooperación, 2001), el modelo de distribución de transporte de carga por carretera para productos (Ortúzar, 2000), los modelos basados en programas integrales de gestión logística (Boto et al., 2005), el multiproducto intermodal modelo de asignación o también llamado transporte combinado o modelo de optimización de red (Márquez, 2008), el modelo logístico de transporte de mercancías con asignación monofuente a multidestino (Mileidy, 2011) y finalmente el modelo de optimización de rutas de transporte utilizando el sistema VRP (Reyes, 2016).

\section{METODOLOGÍA}

Se partió de un diagnóstico sobre la situación actual de la empresa mediante al análisis de la información contenida en los sistemas de información de la compañía: Sistema de Gestión de Posicionamiento Geográfico (GPS), Sistema de Control de Almacenamiento Inteligente (WMS), Intercambio de Documentos Electrónicos (EDI) y el Sistema Tracking de Control de su Operación (SIGELO), una vez visualizada la información, se procedió a definir el sistema de ruteo con cuatro zonas: zona norte, belén, centro y zona loca, mediante el análisis geométrico de los orígenes-destino y las frecuencias de viaje; con este análisis previo, se tomaron los tiempos de cargué, traslado y descargue en cada punto origen-destino; luego, se procedió a implementar con los datos obtenidos un modelo de simulación discreta, previa validación estadística donde se verificó el tipo de distribución para las operaciones de cargue, traslado 
y descargue y se tuvieron en cuenta los estadísticos de Anderson-Darling y KolmogorovSmirnov en el análisis de bondad de ajuste de los datos a la distribución.

Una vez definidas las distribuciones para los tiempos de las operaciones se procedió a construir el modelo con base en los siguientes supuestos:

- Se asume una velocidad media de $18 \mathrm{~km} / \mathrm{h}$.

- Se asume un horario laboral de $10 \mathrm{~h} / \mathrm{día}$.

- Se entregan 500 cajas por día.

- La simulación comienza sin condiciones iniciales.

Finalmente, haciendo uso de la herramienta Promodel se construyó el modelo de simulación discreta para cada zona y se analizaron los resultados arrojados por los mismos.

\section{RESULTADOS}

\section{Diagnóstico situación actual}

La empresa ha integrado tecnología a su operación implementando Sistemas de Gestión de Posicionamiento Geográfico (GPS), Sistemas de Control de Almacenamiento Inteligente (WMS), Intercambio de Documentos Electrónicos (EDI) y Sistema Tracking de Control de su Operación (SIGELO), entre otras herramientas.

Los datos recolectados fueron suministrados por la empresa por medio del software SIGELO, con el cual se tiene mayor control de la operación; esta herramienta arrojó los tiempos de cargue y desplazamiento, que debieron ser organizados para lograr los datos necesarios para la simulación, contándose con una base de datos de 5680 registros, recopilando variables tales como placa del vehículo, fecha, zona, estado del vehículo, responsable y punto de entrega.

Las rutas en sus respectivas zonas: norte, Belén, centro y zona loca, fueron identificadas con la ayuda del Google Maps, tal como se muestra en la Figura1. 


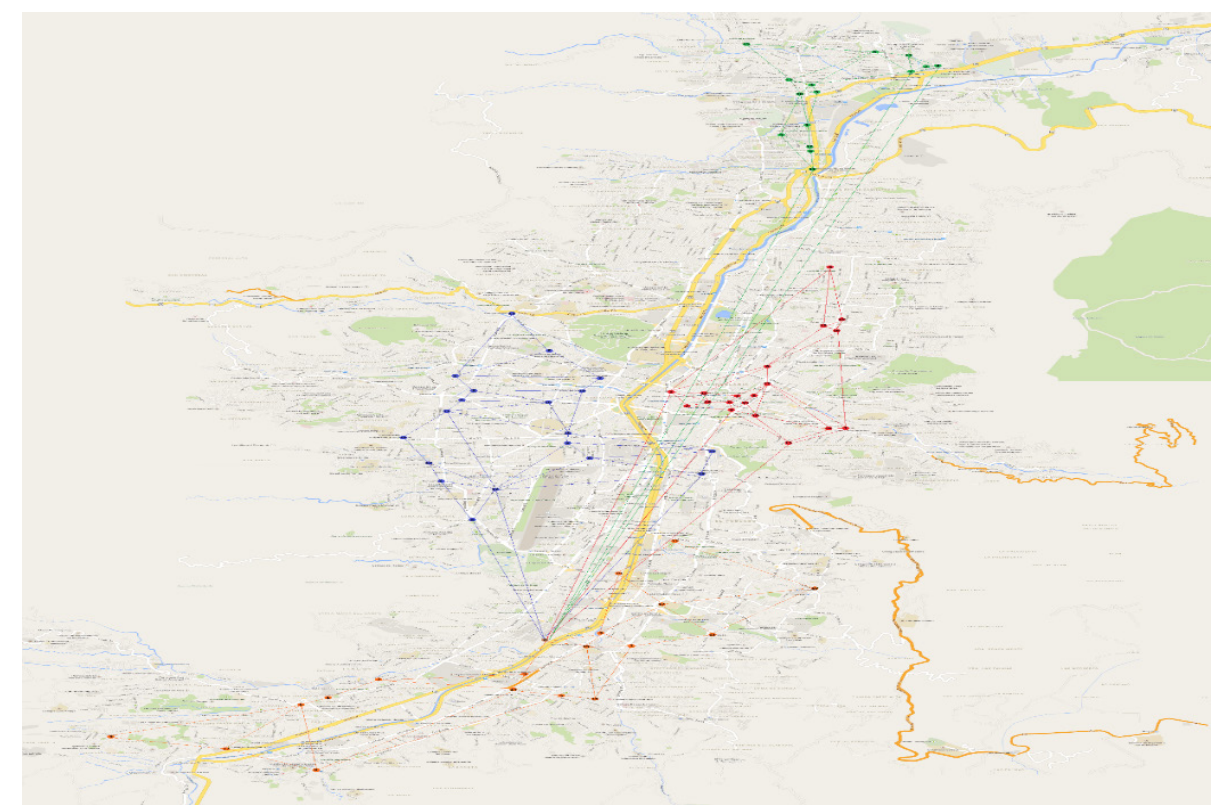

Figura 1. Rutas trazadas para el modelo de simulación intraurbano.

Para el diagnóstico de la situación actual se realizó un análisis descriptivo, mediante la observación de los datos y de las visitas realizadas a la empresa, en las cuales el administrador y los jefes de bodega explicaron la operación que se realiza diariamente.

La Tabla 1 recoge los tiempos de cargue (TC), de recorrido (TR) y de descargue (TD) en promedio por zonas, expresados en minutos, con lo cual se pueden obtener datos acerca de la operación.

Tabla 1. Promedios totales por zona

\begin{tabular}{|c|c|c|c|c|c|}
\hline \multicolumn{2}{|c|}{ TABLA DE PROMEDIOS DE ZONAS } & \multicolumn{3}{|c|}{ TOTAL OPERACIÓN } \\
\hline ZONA & $\begin{array}{c}\text { TC PROM } \\
(\mathrm{min})\end{array}$ & $\begin{array}{c}\text { TR PROM } \\
(\mathrm{min})\end{array}$ & $\begin{array}{c}\text { TD PROM } \\
(\mathrm{min})\end{array}$ & $\begin{array}{c}\text { TOTAL PROM } \\
(\mathrm{min})\end{array}$ & $\begin{array}{c}\text { TOTAL PROM } \\
(\mathrm{h})\end{array}$ \\
\hline BELÉN & 55,10 & 180,5 & 308,6 & 544,2 & 9,1 \\
\hline LOCA & 54,44 & 273,4 & 367,7 & 695,5 & 11,6 \\
\hline CENTRO & 53,78 & 212,8 & 379,4 & 646,0 & 10,8 \\
\hline NORTE & 54,79 & 242,8 & 323,8 & 621,4 & 10,4 \\
\hline TOTAL PROM & 54,5 & 227,4 & 344,9 & 626,8 & 10,4 \\
\hline UTILIZACIÓN $(\%)$ & 8,7 & 36,3 & 55,0 & & \\
\hline
\end{tabular}

Según la tabla anterior, se puede observar que el tiempo de cargue está en el rango estipulado por la empresa, que es un máximo de 60 minutos. Por otro lado, se ve la necesidad de tomar medidas necesarias para el tiempo de descargue, que afecta la operación en un $55 \%$. En cuanto al tiempo de recorrido se observa que se tiene un porcentaje de utilización del $36,27 \%$; se espera que éste sea mejorado con la programación dinámica, la cual genera una ruta óptima para garantizar que el camión cumpla con la demanda en el tiempo de trabajo estipulado por la empresa.

En cada punto de entrega, los auxiliares de bodega deben esperar a que el cliente autorice 
la orden para descargar la mercancía; luego de que esto es aceptado, un auxiliar va desarrumando las cajas y los otros dos van descargando; es este periodo no se ve afectada la operación, sino que se ve afectada cuando es necesario esperar para descargar y al camión le corresponde esperar mientras se descarga y se recibe la factura con la firma del cliente; incluso, algunas veces el conductor debe dejar a los auxiliares en ese punto y continuar el recorrido, para que la operación no se vea tan afectada; sin embargo, esto sí genera un cuello de botella en el sistema debido a que el conductor debe volver por ellos.

\section{Modelo simulado}

Con los datos entregados por la empresa, se procedió a realizar la validación estadística y la construcción del modelo de simulación discreta.

\section{Validación estadística}

Una vez se obtuvo la información, se procedió a la validación estadística, la cual fue realizada por medio de Stat:Fit ${ }^{\circ}$ que es un módulo de Promodel ${ }^{\circledR}$, esto con el fin de saber cuál tipo de distribución se ajusta más a los datos obtenidos.

Para el tiempo de cargue de cada una de las rutas se realizó la prueba de bondad de ajuste, en la cual se aceptan todas las distribuciones, pero se escoge la distribución lognormal por tener el mayor valor de aceptación del rango, lo mismo se hizo para el tiempo de recorrido y de descargue en cada una de las zonas definidas.

A continuación, en la Figura 2, se muestra el resumen de los datos arrojados por Stat::Fit $\circledast$ para el caso ilustrado del tiempo de cargue.

\begin{tabular}{l|l|l} 
Auto :: Fit of Distributions & & \\
\hline Distribution & rank & acceptance \\
\hline & & \\
\hline Lognormal[36,1; 2,94; 0,179$]$ & 100 & do not reject \\
\hline Normal[55,2; 3,38] & 88,8 & do not reject \\
\hline Uniform[50, ; 60,] & 19,1 & do not reject \\
\hline Exponential[50, ; 5,2] & 6,95 & do not reject
\end{tabular}

\begin{tabular}{l|l|l} 
summary & & \\
\hline distribution & $\begin{array}{l}\text { Kolmogorov } \\
\text { Smirnov }\end{array}$ & $\begin{array}{l}\text { Andreson } \\
\text { Darling }\end{array}$ \\
\hline & & \\
\hline Exponential & 0,218 & 1,72 \\
\hline Lognormal & 0,145 & 0,788 \\
\hline Normal & 0,149 & 0,825 \\
\hline Uniform & 0,2 & 1,26
\end{tabular}

Figura 2. Validación Stat:Fit@, tiempo de cargue.

Con las pruebas de Anderson-Darling y Kolmogorov-Smirnov se puedo determinar el grado de ajuste de los datos en los siguientes tipos de distribución: distribución normal, distribución Lognormal, distribución uniforme y distribución exponencial, para las variables medidas de tiempo de cargue, descargue y recorrido. 
En la Tabla 2 se muestra un resumen del tipo de distribución que tiene cada zona en el tiempo de cargue, descargue y recorrido. Las dos columnas de la derecha muestran los valores $p$ para cada una de las pruebas realizadas -Kolmogorov-Smirnov y Anderson-Darling-.

Tabla 2. Resumen de validación de datos

\begin{tabular}{l|l|l|c|c} 
& \multicolumn{1}{|c|}{ TIEMPO } & \multicolumn{1}{c|}{$\begin{array}{c}\text { TIPO DE } \\
\text { DISTRIBUCIÓN }\end{array}$} & $\begin{array}{c}\text { PRUEBA DE } \\
\text { KOLMOGOROV } \\
\text { SMIRNOV }\end{array}$ & $\begin{array}{c}\text { PRUEBA DE } \\
\text { ANDERSON } \\
\text { DARLING }\end{array}$ \\
\hline \multirow{4}{*}{ ZONA BELÉN } & DE CARGUE & LOGNORMAL & 0,619 & 0,490 \\
\cline { 2 - 5 } & DE RECORRIDO & LOGNORMAL & 0,104 & 0,273 \\
\cline { 2 - 5 } & DE DESCARGUE & NORMAL & 0,123 & 0,372 \\
\hline \multirow{4}{*}{ ZONA LOCA } & DE CARGUE & LOGNORMAL & 0,619 & 0,490 \\
\cline { 2 - 5 } & DE RECORRIDO & LOGNORMAL & 0,136 & 0,328 \\
\cline { 2 - 5 } & DE DESCARGUE & NORMAL & 0,105 & 0,399 \\
\hline \multirow{3}{*}{ ZONA NORTE } & DE CARGUE & LOGNORMAL & 0,619 & 0,490 \\
\cline { 2 - 5 } & DE RECORRIDO & LOGNORMAL & 0,086 & 0,170 \\
\cline { 2 - 5 } & DE DESCARGUE & LOGNORMAL & 0,123 & 0,381 \\
\cline { 2 - 5 } & DE CARGUE & LOGNORMAL & 0,619 & 0,490 \\
\cline { 2 - 5 } & DE RECORRIDO & UNIFORME & 0,117 & 0,348 \\
\cline { 2 - 5 } & DE DESCARGUE & NORMAL & 0,185 & 1,090
\end{tabular}

\section{Simulación de Zona Belén}

La Figura 3 muestra los resultados para las locaciones del modelo en la zona Belén simulada por software Promodel®,

\begin{tabular}{|c|c|c|c|c|c|c|c|c|c|c|}
\hline & eneral Locations & Location States Single & Resources & Resource States & Failed Arrivals & Entity Activity & Entity States & & & \\
\hline \multicolumn{11}{|c|}{ ZonaBelen.MOD (Normal Run - Rep. 1) } \\
\hline & Name & Scheduled Time (HR) & Capacity & Total Entries & Avg Time Per & Entry (MIN) & Avg Contents & Maximum Contents & Current Contents & $\%$ Utilization \\
\hline & Icoltrans & 240,00 & 1,00 & 18,00 & & 437,15 & 0.55 & 1,00 & 1,00 & 54,64 \\
\hline & ConsumoBelenPalma & 240,00 & 1,00 & 13,00 & & 502,00 & 0.45 & 1,00 & 0.00 & 45,32 \\
\hline & ConsumoColores & 240,00 & 1,00 & 8,00 & & 575,74 & 0,32 & 1,00 & 1,00 & 31,99 \\
\hline & ConsumoFloresta & 240,00 & 1,00 & 9,00 & & 572,49 & 0,36 & 1,00 & 1,00 & 35,78 \\
\hline & ConsumoLaÁmerica & 240,00 & 1,00 & 10,00 & & 546,46 & 0,38 & 1,00 & 1,00 & 37,95 \\
\hline & ExitoLaAmerica & 240,00 & 1,00 & 9,00 & & 556,48 & 0,35 & 1,00 & 0,00 & 34,78 \\
\hline & ExitoBelen & 240,00 & 1,00 & 13,00 & & 503,09 & 0.45 & 1,00 & 1,00 & 45,42 \\
\hline & ExitoColombia & 240,00 & 1,00 & 7,00 & & 598,98 & 0,29 & 1,00 & 0,00 & 29,12 \\
\hline & ExitoGranvia & 240,00 & 1.00 & 14,00 & & 513,51 & 0,50 & 1,00 & 1,00 & 49,93 \\
\hline & Exito33 & 240,00 & 1,00 & 5,00 & & 516,14 & 0,18 & 1,00 & 1,00 & 17,92 \\
\hline & Exito70 & 240,00 & 1,00 & 7,00 & & 595,15 & 0,29 & 1,00 & 1,00 & 28,93 \\
\hline & ExitoLaureles & 240,00 & 1.00 & 11,00 & & 514,66 & 0,39 & 1,00 & 1,00 & 39,31 \\
\hline & ExitoMolinos & 240,00 & 1,00 & 12,00 & & 551,12 & 0.46 & 1,00 & 1,00 & 45,93 \\
\hline & ExitoRiobledo & 240,00 & 1,00 & 8,00 & & 600,39 & 0,33 & 1,00 & 0,00 & 33,35 \\
\hline & ExitoSanDiego & 240,00 & 1,00 & 4,00 & & 585.45 & 0,16 & 1,00 & 0,00 & 16,26 \\
\hline & ExitoUnicentro & 240,00 & 1,00 & 6,00 & & 523,75 & 0,22 & 1,00 & 1,00 & 21,82 \\
\hline & LaVaquitaLaCastellana & 240,00 & 1,00 & 11,00 & & 521,15 & 0.40 & 1,00 & 0,00 & 39,81 \\
\hline & LaVaquitaBelen & 240,00 & 1,00 & 13,00 & & 526,63 & 0,48 & 1,00 & 0,00 & 47,54 \\
\hline & JumboLa65 & 240,00 & 1,00 & 6,00 & & 589,36 & 0,25 & 1,00 & 0,00 & 24,56 \\
\hline & JumboPremiumPlaza & 240,00 & 1,00 & 4,00 & & 650,64 & 0,18 & 1,00 & 1,00 & 18,07 \\
\hline
\end{tabular}

Figura 3. Resultado de locaciones en Zona Belén por Promodel®

La Figura 4 presenta un histograma en el que se compara la utilización de cada locación, partiendo de las herramientas que ofrece el software Promodel $囚$. Se observó que los clientes con mayor utilización del sistema, es decir, donde se procesan más lento las locaciones, son el Éxito de Gran Vía con un 50\%, La Vaquita de Belén con un 48\% y el Éxito de Molinos con 
un $46 \%$.

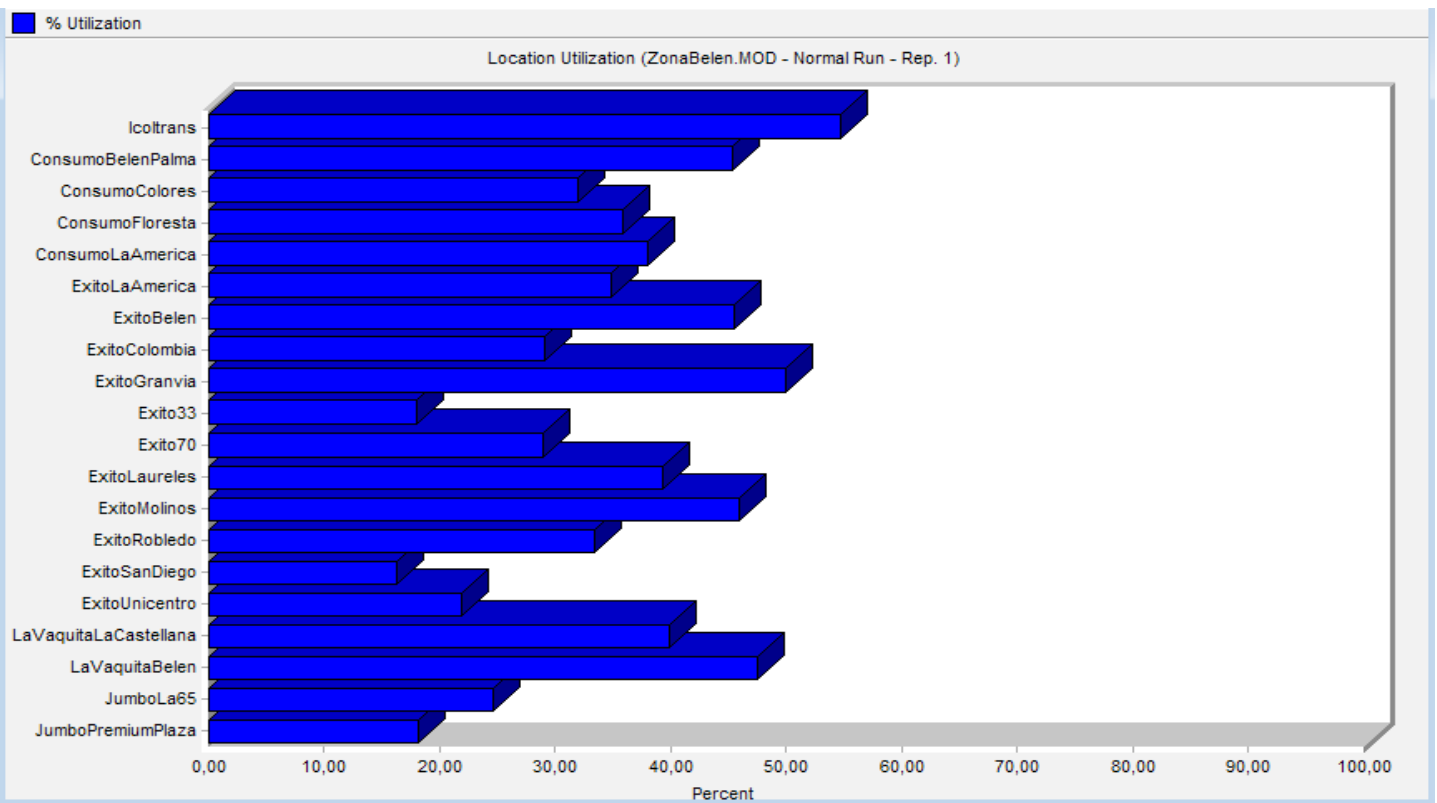

Figura 4. Indicador de utilización en Zona Belén por Promodel ${ }^{\circ}$

En la Figura 5 se muestran los resultados para el recurso del modelo de simulación. Se observó que el recurso (camión) se utilizó en un $96 \%$, lo que indica que con un solo camión se puede satisfacer la demanda diaria y que la disposición de este recurso es indispensable a lo largo de toda la operación.

Name Units Scheduled Time (HR) Number Times Used Avg Time Per Usage (MIN) Avg Time Travel To Use (MIN) Avg Time Travel To Park (MIN) \% Blocked In Travel \% Utilization

\begin{tabular}{lrrrrrr}
\hline Semi Truck & 1,00 & 240,00 & 353,00 & 15,99 & 23,04 & 0,00 \\
\hline
\end{tabular}

Figura 5. Resultado de recursos en Zona Belén por Promodel®.

En la figura 6 se muestran los resultados para la entidad (caja) del modelo. Se analizó que la entidad se mueve durante toda la operación 470 minutos, es decir, cada 7,8 horas de las 9,6 horas de trabajo arrojadas en la simulación; las cajas están en proceso de cargue o descargue, lo que implica que las cajas están en proceso el $81 \%$ de toda la operación.

De igual forma se procedió para las otras zonas del modelo. 


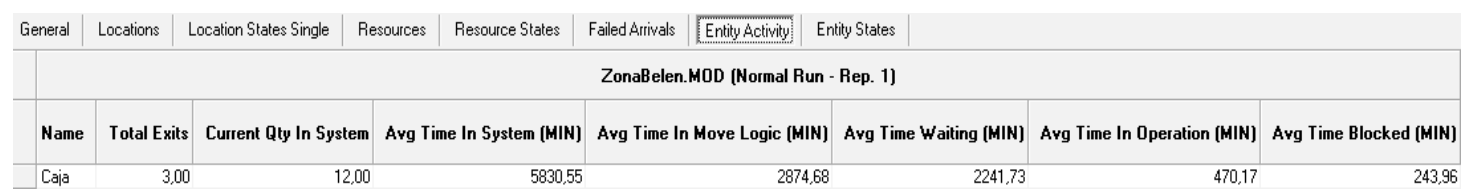

Figura 6. Resultado actividad de la locación en Zona Belén por Promodel®.

\section{Simulación de Zona Loca}

En cuanto a las locaciones, para esta zona se programó en la simulación un tiempo de 310 horas, el cual se asignó por los días trabajados y el tiempo promedio (tiempo total de ciclo). Cabe destacar que donde hay un mayor promedio de tiempo de operación es en la locación del Éxito de El Poblado y que el Consumo de Itagüí es donde el proceso se detiene la menor cantidad de tiempo. Asimismo, se observó que los clientes con mayor utilización del sistema, es decir, donde se procesan más lento las locaciones son Jumbo Las Vegas, 47\%, Almacenes Marion, 47\%, y Easy Colombia, con un 45\%.

En lo concerniente a los recursos, se observó que el recurso (camión) se utilizó en un 94\%, lo que indica que con un solo camión se puede satisfacer la demanda diaria y que la disposición de este recurso es indispensable a lo largo de toda la operación.

Del mismo modo, se analizó que la entidad se mueve durante toda la operación 424 minutos, es decir, cada 7,1 horas de las 9,4 horas de trabajo arrojadas en la simulación; las cajas están en proceso de cargue o descargue, lo que implica que las cajas están en proceso el $76 \%$ de toda la operación.

\section{Simulación de Zona Centro}

Con respecto a las locaciones, para esta zona se programó en la simulación un tiempo de 290 horas, el cual se asignó por los días trabajados y el tiempo promedio (tiempo total de ciclo). Cabe destacar que donde hay un mayor promedio de tiempo de operación es en la locación Éxito de San Antonio y que Olímpica de Villa Nueva es donde el proceso se detiene la menor cantidad de tiempo. Adicionalmente, se observó que los clientes con mayor utilización del sistema, es decir, donde se procesan más lento las locaciones, son Pasteur La Torre, con un $40 \%$, Pasteur de Ayacucho, con un 38\%, y Almacén Multielectro, con un 37\%.

El recurso (camión) se utilizó en un $91 \%$, lo que indica que con un solo camión se puede satisfacer la demanda diaria y que la disposición de este recurso es indispensable a lo largo de toda la operación.

Además, la entidad se mueve durante toda la operación 475 minutos, es decir, cada 7,9 horas de las 9,1 horas de trabajo arrojadas en la simulación; las cajas están en proceso de cargue o descargue, lo que implica que las cajas están en proceso el $87 \%$ de toda la operación.

\section{Simulación de Zona Norte}

Para esta zona se programó en la simulación un tiempo de 280 horas, el cual se asignó por los días trabajados y el tiempo promedio (tiempo total de ciclo). Cabe destacar que donde hay un mayor promedio de tiempo de operación es en la locación de Superahorro Bello y que el Éxito de Niquía es donde el proceso se detiene la menor cantidad de tiempo. Se observó que los clientes con mayor utilización del sistema, es decir, donde se procesan más lento las locaciones, son Cipa S.A., con un 55\%, Jumbo de Bello, también con un 55\%, y el Consumo de Bello, con un 53\%. 
En esta zona el recurso (camión) se utilizó en un 97\%, lo que indica que con un solo camión se puede satisfacer la demanda diaria y que la disposición de este recurso es indispensable a lo largo de toda la operación.

Finalmente, se analizó que la entidad se mueve durante toda la operación 379 minutos, es decir, cada 6,3 horas de las 9,7 horas de trabajo arrojadas en la simulación; las cajas están en proceso de cargue o descargue, lo que implica que las cajas están en proceso el $65 \%$ de toda la operación.

\section{Comparación modelo actual vs modelo final}

Para esta comparación se realizó la Tabla 3, que incluye los ciclos totales de cada operación y el porcentaje de mejora obtenido por la optimización de cada ruta. Para cada zona, los tiempos del modelo final se ajustan a lo esperado (cumplir con las diez horas de trabajo) que en el modelo actual, por lo cual cabe suponer que la implementación de este modelo, aunque no llegue a tener la precisión que se obtiene con la simulación, sí podría contribuir a que los procesos sean óptimos, llegando, en el mejor de los casos, a mejorar el rendimiento casi en un 30\% como promedio o incluso cerca del $40 \%$ para las zonas Loca y Norte, dichos promedios permiten con respeto a estudios previos garantizar unos tiempos estándar para las operaciones de cargue, traslado y descargue que permitan planificar a futuro las rutas de una manera óptima.

El porcentaje optimizado en la operación de distribución -29,66\% (Tabla 3), se debe en gran medida a la eliminación de tiempos de espera en cada cliente de la ciudad. En adición se destacaron en la simulación escenarios corridos a lo largo del tiempo, sin presentarse ningún tipo de urgencias, lo cual implicaría un aumento de tiempo en la operación diaria.

Una de las variables, de gran impacto para la eficiencia de los recorridos, se refiere a los altos tiempos de espera que deben afrontar los camiones cuando van a entregar las mercancías, puesto que en algunas ocasiones deben esperar hasta 30 minutos mientras el cliente recibe y firma a conformidad la factura de entrega. Este tiempo se puede convertir en un cuello de botella para la operación por cuanto el camión permanece quieto en unos minutos que podría aprovechar para otro desplazamiento o para procesos de cargue y descargue.

Tabla 3. Tabla comparativa de modelo actual vs propuesto

\begin{tabular}{|c|c|c|c|c|c|}
\hline \multicolumn{5}{|c|}{ TABLA COMPARATIVA } & \multirow[b]{3}{*}{$\begin{array}{c}\% \\
\text { MEJORA }\end{array}$} \\
\hline \multirow[b]{2}{*}{ ZONA } & \multicolumn{2}{|l|}{ MODELO ACTUAL } & \multicolumn{2}{|l|}{ MODELO FINAL } & \\
\hline & $\begin{array}{c}\text { TOTAL } \\
\text { PROMEDIO(min) }\end{array}$ & $\begin{array}{c}\text { TOTAL } \\
\text { PROMEDIO(h) }\end{array}$ & $\begin{array}{c}\text { TOTAL } \\
\text { PROMEDIO(min) }\end{array}$ & $\begin{array}{c}\text { TOTAL } \\
\text { PROMEDIO(h) }\end{array}$ & \\
\hline ZONA BELÉN & 544,2 & 9,1 & 470,0 & 7,8 & 13,63 \\
\hline ZONA LOCA & 695,5 & 11,6 & 424,0 & 7,1 & 39,04 \\
\hline $\begin{array}{l}\text { ZONA } \\
\text { CENTRO }\end{array}$ & 646,0 & 10,8 & 474,0 & 7,9 & 26,63 \\
\hline $\begin{array}{l}\text { ZONA } \\
\text { NORTE }\end{array}$ & 621,4 & 10,4 & 377,0 & 6,3 & 39,33 \\
\hline TOTAL PROM & 626,8 & 10,4 & 436,3 & 7,3 & 29,66 \\
\hline
\end{tabular}




\section{CONCLUSIONES}

Los hallazgos de investigación evidencian la necesidad de implementar un software que permita programar toda la operación. Es necesario el uso de tecnología desde que se hace la separación, cargue y descargue de la mercancía, dejando a un lado el empirismo y logrando control y manejo de la operación.

Los resultados, permiten afirmar que las herramientas tecnológicas disminuyen el margen de error y colaboran a tomar mejores decisiones a la hora de programar las rutas de entrega, la distribución de las mercancías y demás variables que puedan influir en procesos distribución de mercancías.

\section{REFERENCIAS}

BOTO GIRALDA, et al. Sistema Inteligente para la Gestión Dinámica de Flotas de Transporte. En: Actas XII Seminario Anual de Automática, Electrónica Industrial e Instrumentación, pp. 643-648. Madrid, 2005.

CAPLICE, C. Transportation Management Operational Networks. ESD.260/15.770/1.260. Logistics Systems. MIT Center for Transportation \& Logistics, 2006. Disponible en: https:// ocw.mit.edu/courses/engineering-systems-division/esd-260j-logistics-systems-fall-2006/ lecture-notes/lect21.pdf

CARRANZA, O., y SABRIA, F. Logística: mejores prácticas en Latinoamérica. México D.F., México: International Thomson Editores. 2005.

FERREL, O, et al. Introducción a los Negocios en un Mundo Cambiante, Cuarta Edición, México., Mc Graw Hill, 2004

LAMB Ch., HAIR J. y MC DANIELI C. Marketing, Sexta Edición, México, International Thomson Editores S.A., 2002

MARQUÉZ, L. Modelo de oferta de transporte para Colombia: Calibración y Asignación. Unión Temporal Modelación de Transporte. Bogotá: Ministerio de Transporte, 2008.

MILEYDI. P. Modelado de Sistemas de Transporte Masivo Empleando Dinámica de Sistemas: Caso Transmilenio S.A. En: IX Congreso Chileno de Investigación Operativa, OPTIMA. Universidad de La Frontera, Temuco. Chile. 2011.

Ministerio de Planificación y Cooperación de Chile. MIDEPLAN. Manual de operación del modelo ESTRAUS, Versión 2.04, Santiago, Chile. 2001.

Ministerio de Transporte Colombia. Modelo para el observatorio de carga. 2009. Disponible en: https://www.mintransporte.gov.co/loader. php?IServicio=Tools2\&ITipo=descargas\&IFuncion=descargar\&id=1214 2009 .

ORTUZAR, J. Modelos de Demanda de Transporte. $2^{\text {a }}$ Edición, México. Ediciones Universidad Católica de Chile. Alfaomega. 2000. 
REYES, N. Modelo de optimización de programación de rutas para una empresa logística peruana usando herramientas FSMVRPTW. Perú. Revista Industrial Data, 2016, v. 19, n.2, pp.118-123

ROBUSTÉ, A.F. E-logistics. Barcelona. Editorial UPC. 2005. 